- Describes the prevalence and possible aetiological factors of hypodontia.

- Highlights the role of genetic factors in tooth development and agenesis.

- Emphasises the importance of multidisciplinary approach in the management of complex cases of hypodontia.

\title{
Severe hypodontia in a set of triplets
}

\author{
S. O. Adeboye, ${ }^{1}$ B. O. I. Cole, ${ }^{2}$ R. S. Hobson, ${ }^{3}$ M. J. Wright ${ }^{4}$
}

\begin{abstract}
Hypodontia is the developmental absence of one or more teeth from the dentition and constitutes one of the most common developmental anomalies in humans with a reported prevalence of 1.6 to $9.6 \%$ in the permanent dentition. Hypodontia may occur in association with other genetic diseases, or as an isolated familial or sporadic form. This article describes the rare phenomenon of severe hypodontia in a set of triplets. The triplets presented with congenital absence of the second molars, second premolars in all quadrants and lower central incisors. An additional five teeth (upper canines, upper lateral incisors and upper left first premolar) were missing in one of the triplets. The treatment plan and the possible genetic mode of inheritance are discussed.
\end{abstract}

\section{INTRODUCTION}

Hypodontia is the developmental absence of one or more teeth from the dentition and constitutes one of the most common developmental anomalies in humans., ${ }^{1,2}$ Other frequently used terms are Oligodontia, partial anodontia and anodontia. Oligodontia describes the absence of multiple teeth usually in association with specific syndromic diagnoses or severe abnormalities. ${ }^{3}$ Anodontia, the total absence of teeth, is rare, and usually associated with hypohidrotic ectodermal dysplasia, a condition inherited in an X-linked recessive manner. ${ }^{1}$ The term partial anodontia is contradictory and no longer used. Hypodontia presents with varying degrees of severity defined by the number of missing

\footnotetext{
1*-3Department of Child Dental Health, Newcastle Dental Hospital and School; ${ }^{4}$ Department of Clinical Genetics, Royal Victoria Infirmary, Newcastle-upon-Tyne

*Correspondence to: Mrs Solape Adeboye, Department of Child Dental Health, Newcastle Dental Hospital,

Richardson Road, Newcastle NE2 4AZ

Email:solape.adeboye@nuth.nhs.uk
}

\section{Refereed Paper}

Accepted 16 February 2006

DOI: $10.1038 /$ sj.bdj.4813818

๑ British Dental Journal 2006; 201: 93-96 teeth. It is described as mild when one or two teeth are congenitally missing, moderate when three to five teeth are missing. The absence of six or more teeth, excluding third molars, is classified as severe hypodontia..$^{4,5}$

Hypodontia may occur in association with other genetic diseases, or as an isolated familial or sporadic form and frequently affects the permanent rather than the primary dentition. ${ }^{4,6}$ The prevalence in the primary dentition is less than $1 \%$ of the population, and most frequently involves the mandibular incisors. ${ }^{1}$

The teeth most frequently affected in the permanent dentition are the third molars, second premolars and lateral incisors; the absence of first and second molars is described as rare. ${ }^{7}$ Reported prevalence of hypodontia in the permanent dentition varies from 1.6\% to $9.6 \%$ in the population, excluding third molars which occurs in up to $25 \%$ of the population. ${ }^{6}$ The variation in prevalence is attributed to differences in sampling techniques, diagnostic criteria and racial derivation of the population studied. ${ }^{8,9}$ The most frequently affected teeth also vary with the population type; in Asians, the mandibular incisors are the most frequently missing teeth, ${ }^{9}$ in African Americans it is the mandibular second premolars ${ }^{10}$ and in Caucasians, it is the maxillary lateral incisor and mandibular second premolars. ${ }^{6}$ A previous report suggests females are affected more often than males, with an approximate ratio of $3: 2 .^{11}$

A number of possible aetiological factors have been suggested. These include hereditary factors, association with systemic syndromes and conditions, environmental factors, such as trauma, infections during pregnancy and early childhood, hormonal dysfunction, radiation therapy, chemotherapeutic medications and evolutionary trend towards fewer teeth. ${ }^{6,12}$ The nature of genetic influence is complex and the mode of inheritance in many families is unclear. Various modes of inheritance have been reported, including autosomal-dominant, ${ }^{7}$ autosomal recessive ${ }^{13}$ and $\mathrm{X}$ linked recessive. ${ }^{14}$ Polygenic and multifactorial models have also been proposed with expression of hypodontia being influenced by modifying genes and environmental factors. ${ }^{6,12,15}$

Cases of hypodontia in family members and twins have been studied to 


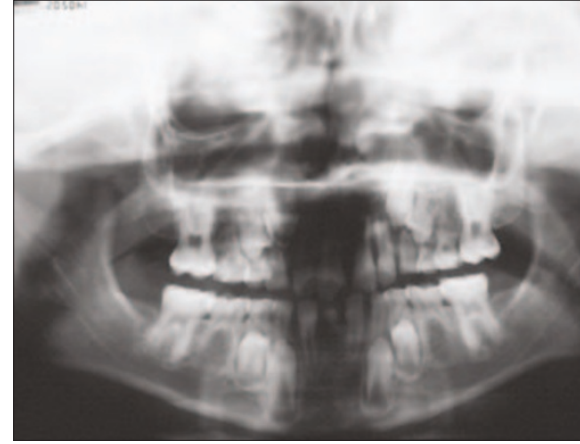

Fig. 1 Panoramic radiograph of Case 1

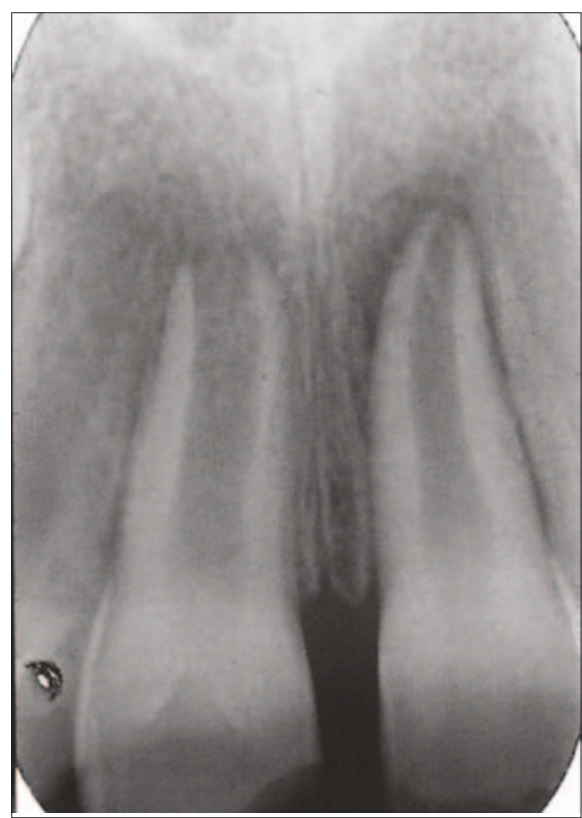

Fig. 2 Periapical radiograph of maxillary central incisors of Case 1

identify the genetic link and possible mode of inheritance. ${ }^{16-19}$ Three articles on hypodontia in triplets ${ }^{18,20,21}$ were found using a PubMed search and none described hypodontia of the severity seen in the patients in this case report. This article describes a case involving a set of triplets with severe hypodontia, affecting molars, premolars and incisors.

\section{CASE REPORT}

A set of male triplets aged 10 years were referred by their local practitioner to the interdisciplinary hypodontia clinic in our dental hospital as a result of missing teeth in the permanent dentition. Patients are identified as Cases 1,2 and 3. Cases 1 and 2 were identical in appearance and of similar height. Case 3 had little facial resemblance to his brothers and was shorter in stature. Their skin and hair appeared clinically normal. The family history was positive for missing teeth in the father, but the family declined to give detailed information on the extended family. They also declined further genetic investigation, counselling and use of clinical photographs.

\section{Case 1}

The patient was fit and well with a history of asthma as an infant. There was no other history of systemic illnesses or medical congenital abnormalities reported.

Clinical examination revealed the patient to have facial symmetry and be in mixed dentition. The erupted permanent teeth present were: UR6, UR2, UR1, UL1, UL2, UL6, LL6, LL2, LR2 and LR6. The primary teeth present were: URE, URD, URC, ULC, ULD, ULE, LLE, LLD, LLC, LLA, LRC, LRD and LRE.

The upper right central incisor (11) had an enamel-dentine fracture sustained about a year prior to the first visit, following a bicycle accident. Labial sinuses were present in relation to upper right and left central incisors (11 and 21), which tested negative to sensibility tests. He presented with molar Class II relationship and incisal Class II div 1. The oral hygiene was good with no carious lesion of the teeth.

Radiographic examination (Fig. 1) showed the following teeth were congenitally missing: UR8, UR7, UR5, UL5, UL7, UL8, LL8, LL7, LL5, LL1, LR1, LR5, LR7 and LR8.

A periapical radiograph (Fig. 2) of UR1 (11) and UL1 (21) revealed incomplete root development and a wide open apex of UR1. The UL1 showed an apex with apparent complete root development. There was a large radiolucency above both apices. The crown of the UR1 had an oblique fracture into dentine and a possible dens invaginatus.

\section{Case 2}

Case 2 was medically fit with a history of adenoidectomy and tonsillectomy at age six years.

Clinical examination revealed facial symmetry; the patient was in mixed dentition with good oral hygiene and no carious lesions. The following permanent teeth were present and erupted: UR6, UR2, UR1, UL1, UL2, UL6, LL6, LL2, LR2 and LR6. The primary teeth present were: URE, URD, URC, ULC, ULD, ULE, LLE, LLD, LLC, LRC, LRD and LRE. The occlusion was molar Class II relationship and incisal Class II div 1.

Radiographic examination (Fig. 3) confirmed that UR8, UR7, UR5, UL5, UL7, UL8, LL8, LL7, LL5, LL1, LR1, LR5, LR7 and LR8 were congenitally missing.

\section{Case 3}

This patient was medically fit and well with no history of childhood illnesses. Clinical examination revealed facial symmetry and a mixed dentition with the following permanent teeth: UR6, UR1, UL1, UL6, LL6, LL2, LR2, LR4 and LR6.

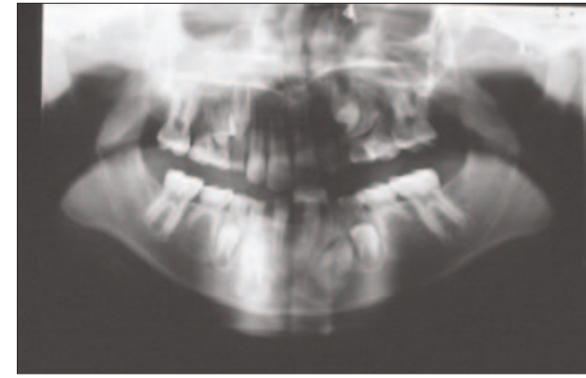

Fig. 3 Panoramic radiograph of Case 2

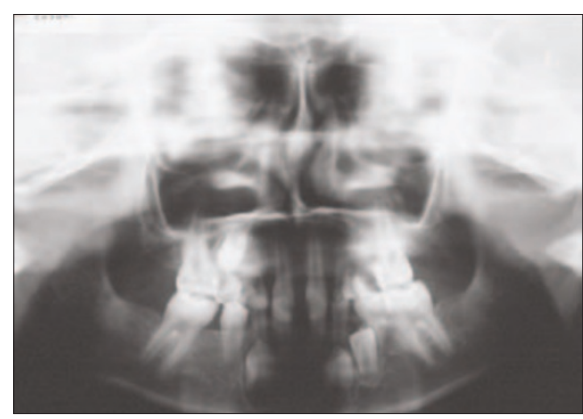

Fig. 4 Panoramic radiograph of Case 3

The primary teeth present were: URE, URD, URC, ULC, ULD, ULE, LLE, LLC and LRC. The patient's oral hygiene was good and UR6, UL6, ULD, ULE, LRC were carious.

Radiographic examination (Fig. 4) confirmed congenitally missing teeth to be: UR8, UR7, UR5, UR3, UR2, UL2, UL3, UL4, UL5, UL7, UL8, LL8, LL7, LL5, LL1, LR1, LR5, LR7 and LR8.

\section{TREATMENT PLAN}

At the time of the first visit to the hypodontia clinic, the triplets considered their appearance and ability to eat satisfactory. The patients' immediate dental needs were assessed and collaborative treatment embarked upon at the dental hospital and the local dental practitioner. The need to maintain good oral hygiene was emphasised.

Aggressive preventive measures were instituted in the triplets in the form of oral hygiene instructions, dietary advice based on a three-day diet history chart and the use of fluoride and fissure sealants where appropriate. These, along with regular review appointments, were important aids to prevent the loss of the natural teeth while awaiting exfoliation of deciduous teeth and further eruption of the permanent teeth.

Root canal treatment on upper right and left central incisors (UR1, UL1) was initiated for Case 1. The teeth were dressed with non-setting calcium hydroxide paste (Ultracal ${ }^{\circledR}$ XS, Ultradent products, Inc, 505 West 10200 South, South Jordan, Utah 84095, USA) every four months to rid the teeth of apical infection and achieve apical closure before 
obturation of the canals with gutta percha. UL1 was obturated after four months of calcium hydroxide dressing. With UR1, a longer period of 14 months was required to achieve good apical seal. Immediate apical closure using Mineral Trioxide Aggregate (MTA) was ruled out in order to control the infection in the teeth and the apical region. ${ }^{22,23}$ Case 2 was referred back to the dental practitioner for restoration of the carious teeth and maintenance of his preventive measures. Case 3 required no restorative work apart from routine preventive measures. Further review appointment on the hypodontia clinic was arranged for the definitive treatment plan, following exfoliation of the primary teeth and eruption of the canines and the premolars.

The principal aim of treatment in cases of hypodontia is to replace missing teeth and bone and hence improve the patient's appearance, speech, and masticatory efficiency. ${ }^{4}$ The importance of a team approach in the management of complex cases referred to the hypodontia clinic cannot be overemphasised, as this ensures the early involvement of orthodontics, paediatric dentistry, restorative dentistry and oral surgery. Close liaison and often, simultaneous participation in treatment by each team member, ensures appropriate timing and management of each stage of treatment. The general dental practitioner with the appropriate skills can also provide or contribute to the high quality care for these patients and fulfil a number of key roles in patient care. ${ }^{24} \mathrm{An}$ over-riding priority is the maintenance and retention of the natural teeth for as long as possible.

\section{DISCUSSION}

This case report describes severe hypodontia in a set of triplets with no evidence of a syndromic cause for the hypodontia. There was a known family history of missing teeth on the paternal side, but unfortunately the family did not consent to further genetic investigation and counselling. The triplets presented with congenital absence of the second molars, second premolars in all quadrants and the lower central incisors (10 teeth excluding the third molars). Case 3 had an additional five teeth missing (upper canines, upper lateral incisors and upper left first premolar). The discrepancy in the number of deciduous teeth present in the triplets was due to early loss in some instances. It was interesting to note that the pattern of missing teeth were similar in Cases 1 and 2 who were very similar in physical and facial appearance. Unfortunately the family did not consent to zygosity studies on the triplets. Triplet pregnancies can either be monozygous, dizygous or trizygous.

A number of family and twin studies investigating the possible role of genetic and environmental factors in hypodontia have been reported. A previous study found no significant difference in the frequencies of concordance and discordance for missing teeth between monozygotic and dizygotic twins, suggesting that heritable factors were less likely to be important. They also reported a higher incidence of hypodontia in premature children compared to full-term children, further highlighting the possible role of an environmental factor. ${ }^{25}$ Contrary to this, another study described a high degree of similarity in the dental features and pattern of malocclusion in a set of monozygotic triplets, indicating a genetic cause. Two of the triplets presented with mirror image congenital absence of the lower second premolars, which were all present in the third child. The authors suggested the discordance between the triplets was due to a difference in a gene mutation during the process of gene recombination or to a difference in gene expressivity. ${ }^{20}$ Möller et al. ${ }^{21}$ described a mild case of familial hypodontia in a set monozygotic triplets and their mother. All affected members had different phenotypic expression of the trait. Since the triplets in the study ${ }^{21}$ were confirmed to be genetically monozygotic, they concluded that the triplets demonstrated non-genetic variation in the expression of familial hypodontia. Another report ${ }^{18}$ demonstrated concordance for hypodontia and/or hypoplasia of the maxillary lateral incisors in monozygotic members and discordance in dizygotic members in twins and triplets studies which strongly indicated a dominant genetic aetiology. These reports described cases of mild hypodontia, the extent of which does not compare with the severity of our cases.

Although there was a positive history of hypodontia in the father, the pattern of missing teeth was not known and could not be commented on.

Severe hypodontia (six or more congenitally missing teeth) are found only in about one in 15 hypodontia patients. ${ }^{4}$ The prevalence of hypodontia has also been shown to be higher within the extended family circle than in the general population, which supports a hereditary tendency and the proportion of relatives affected varied with the severity of the condition. ${ }^{17}$ A study of children with missing teeth found that up to half of their siblings or parents also had missing teeth compared with the rate of $6 \%$ found in the general population. ${ }^{26} \mathrm{~A}$ recent study reported a lower prevalence of missing teeth to be $22 \%$ in first-degree relatives of patients compared to $4.4 \%$ in the general population. ${ }^{27}$

Investigation of the role of genetic factors in mouse models indicates that tooth development is regulated by interactions between epithelial and mesenchymal cells and is dependent on a number of genes. The genes encode transcription factors and signal molecules that exhibit dynamic expression patterns during embryogenesis in a variety of tissues, and play an important role in the formation of a number of organs, including the teeth. ${ }^{28,29}$

In humans, mutations in homeobox gene Msx1 and the paired box gene Pax9 have been associated with selective tooth agenesis. ${ }^{15,30}$ Mutation in Msx1 can also cause orofacial clefting. ${ }^{31,32}$ Mutations in Pax9 have been associated with unique phenotypes of familial tooth agenesis that mainly involve posterior teeth. ${ }^{15,33}$ Heterozygous deletion of the entire Pax 9 gene has also been reported in severe hypodontia involving the primary and permanent molars in a small nuclear family. ${ }^{19}$

The absence of mutations in the coding regions of either Pax9 or Msx1, in some patients with oligodontia indicated that other genes encoding transcription factors might be responsible for the patterning of the dentition. ${ }^{15}$ A study of non-syndromic oligodontia in a multigenerational family detected no mutation within the coding region of Msx1, though total exclusion of this locus was not carried out. The homeobox genes encoding the transcription factors - Barx1, 0tlx2, Lhx6, Lhx7, Dlx1, Dlx2, Pax9 and Lef1 - that influence the early specification of odontogenic mesenchyme and the patterning of dentition, were listed as strong candidate genes for this type of oligodontia and other forms of oligodontia. ${ }^{7}$ Diffusible factors like FGF8 and BMP4 were also likely candidate genes in the underlying pathogenesis that leads to the failure of tooth development, as they had been shown to interact in either a synergistic or an antagonistic manner with several master transcription factors that influence tooth formation. ${ }^{29,34}$

There is an ongoing effort to understand at the genetic molecular level, the role of several factors in the aetiology of tooth agenesis and orofacial clefting. Recent papers ${ }^{31,32}$ have opened up further avenues for research at the genetic molecular level. The genetic aetiology of tooth agenesis is almost certainly heterogeneous given the different mutations identified in Msx1 and Pax9 genes, which are associated with a similar pattern of selective tooth agenesis. Other genes yet 
to be discovered may be responsible in cases where these two genes and other factors have been excluded.

\section{CONCLUSION}

The triplets showed a very high degree of similarity in the pattern of hypodontia, supporting a strong genetic link. There was a positive family history in the paternal side of the family, but unfortunately this could not be followed up. The triplets demonstrated concordance in the pattern and number of missing permanent teeth (10 teeth, excluding the third molars) in two of the boys, but the third triplet was discordant for the additional five missing teeth. We could assume this to be the result of gene mutation or difference in gene expressivity as considered in earlier studies. The extent of the role of environmental factors though may not be known, but could not be completely ruled out.

1. Neville B W, Damm D D, Allen C M, Bouquot J E. Oral and maxillofacial pathology. pp 60-61. Philadelphia: WB Saunders, 1995.

2. Symons A L, Stritzel F, Stamatiou J. Anomalies associated with hypodontia of the permanent lateral incisors and second premolar. J Clin Pediatr Dent 1993; 17: 109-111.

3. Stewart R E, Barner TK, Troutman K C. Wei SHY Pediatric dentistry, scientific foundations and clinical practice. pp 87-109. St. Louis: CV Mosby, 1982.

4. Hobkirk J A, Brook A H. The management of patients with severe hypodontia. J Oral Rehabil 1980; 7: 289-298.

5. Hobkirk J A, King P A, Goodman J R, Jones S P. Hypodontia: 2. The management of severe hypodontia. Dent Update 1995; 22: 8-11.

6. Graber $L$ W. Congenital absence of teeth: a review with emphasis on inheritance patterns. J Am Dent Assoc 1978: 96: 266-275.

7. Goldenberg M, Das P, Messersmith M, Stockton D W et al. Clinical, radiographic, and genetic evaluation of a novel form of autosomal-dominant oligodontia. J Dent Res 2000; 79: 1469-1475.

8. Jorgenson R J. Clinician's view of hypodontia. JAm Dent Assoc 1980; 101: 283-286.

9. Davis P J. Hypodontia and hyperdontia of permanent teeth in Hong Kong schoolchildren. Comm Dent Oral Epidemiol 1987; 15: 218-220.

10. Silinas $C F$, Jorgenson R J. Dental anomalies in a black population. J Dent Res 1974; 53: 237.

11. Chai W L, Ngeow W C. Familial cases of missing mandibular incisor: Three case presentations. Dent Update 1999; 26: 298-302.

12. Lyngstadaas S P, Nordbø, Gedde-Dahl Jr T, Thrane P S. On the genesis of hypodontia and microdontia: synergism or allelism of major genes in a family with six affected members. J Med Genet 1996; 33: 137-142.

13. Pirinen $S$, Kentala A, Nieminen P et al. Recessively inherited lower incisor hypodontia. J Med Genet 2001: 38: 551-556.

14. Erpenstein $H$, Pfeiffer $R A$. Sex-linked dominant hereditary reduction in number of teeth. Humangenetik 1967: 4: 280-293.

15. Mostowska A, Kobielak A, Biedziak B, TrZeciak W H. Novel mutation in the paired box sequence of PAXg gene in a sporadic form of oligodontia. Eur J Oral Sci 2003; 111: 272-276

16. Markovic M. Hypodontia in twins. Swed Dent J 1982 Suppl. 15: 153-162.

17. Brook A H. A unifying aetiological explanation for anomalies of human tooth number and size. Arch Oral Biol 1984: 29: 373-378.

18. Markovic M D. At the crossroads of oral facial genetics. Europ J Orthodont 1992; 14: 469-481.

19. Das $P$, Hai $M$, Elcock $C$ et al. Novel missense mutations and a 288-bp exonic insertion in PAX9 in families with autosomal dominant hypodontia. Am J Med Genet 2003: 118A: 35-42.

20. Markovic M, Trisovic D. Monozygotic triplets with discordance for some traits. Europ J Orthodont 1979; 1: 189-192.

21. Möller P, Berg K, Ruud A F, Kvein T K. Variable expression of familial hypodontia in monozygotic triplets. Scand J Dent Res 1981; 89: 16-18.

22. Giuliani V, Baccetti T, Pace R, Pagavino G. The use of MTA in teeth with necrotic pulps and open apices. Dent Traumatol 2002; 18: 217-221.

23. Maroto M, Barberia E, Planells P, Vera V. Treatment of a non-vital immature incisor with mineral trioxide aggregate (MTA). Dent Traumato/ 2003; 19: 165-169.

24. Hobson R S, Carter N E, Gillgrass T J et al. The interdisciplinary management of hypodontia: the relationship between an interdisciplinary team and the general dental practitioner. Br Dent J 2003; 194: 479-482.

25. Boruchov M J, Green LJ. Hypodontia in twins and families. Am J Orthod 1971; 60: 165-174

26. Grahnen $\mathrm{H}$. Hypodontia in the permanent dentition. A clinical and genetical investigation. Odontol Rev 1956; 7: 1-100.

27. Brook $\mathrm{A} H$, Elcock $\mathrm{C}, \mathrm{Al}$-Sharod $\mathrm{M} \mathrm{H}$ et al. Further studies of a model for the etiology of anomalies of tooth number and size in humans. Conn Tiss Res 2002; 43: 289-295.

28. Thesleff I. The genetic basis of normal and abnormal craniofacial development. Acta Odontol Scand 1998; 56: 321-325.

29. Peter $H$, Balling R. Teeth: where and how to make them. Trends Genet 1999; 15: 59-65.

30. Vastardis $\mathrm{H}$. The genetics of human tooth agenesis: new discoveries for understanding dental anomalies. Am J Orthod Dentofac Orthop 2000; 117: 650-656.

31. Murray J C, Schutte B C. Cleft palate: players, pathways, and pursuits. J Clin Inves 2004; 113 1676-1678

32. Rice $\mathrm{R}$, Spencer-Dene $B$, Connor E C et al. J Clin Inves 2004: 113: 1692-1700.

33. Mensah J K, Ogawa T, Kapadia H et al. Functional Analysis of a mutation in PAX9 associated with familial tooth agenesis in humans. J Bio/ Chem 2004; 279: 5924-5933.

34. Tucker A S, Sharpe PT. Molecular genetics of tooth morphogenesis and patterning: the right shape in the right place. J Dent Res 1999; 78: 826-834. 